\title{
Triple Gene Analysis Using Samples Obtained by Endobronchial Ultrasound-guided Transbronchial Needle Aspiration
}

\author{
Kyungjong Lee ${ }^{1}$, Sang-Won Um ${ }^{1}$, Byeong-Ho Jeong ${ }^{1}$, Jung Wook Yang ${ }^{2}$, \\ Yoon-La Choi ${ }^{2}$, Joungho $\mathrm{Han}^{2}$, Hojoong $\mathrm{Kim}^{1}{ }^{1}$ and O Jung Kwon ${ }^{1}$
}

\begin{abstract}
Objective A mutational analysis of tumor tissue samples is an important part of advanced lung cancer treatment strategies. This study evaluated the efficacy of a triple gene analysis using samples obtained via endobronchial ultrasound-guided transbronchial needle aspiration (EBUS-TBNA).

Methods Either metastatic lymph nodes or primary lung mass samples obtained by EBUS-TBNA were collected between May 2011 and May 2013. We consecutively analyzed epidermal growth factor receptor $(E G F R)$, V-Ki-ras2 Kirsten rat sarcoma viral oncogene homolog (KRAS), and anaplastic lymphoma kinase $(A L K)$ fusion genes using remnant tissue samples.

Results A total of 109 patients were diagnosed with non-small cell lung cancer (NSCLC). Of these, 70\% were adenocarcinoma, 27\% squamous cell carcinoma with NSCLC, and 3\% were related to other types of lung cancer. EGFR mutations were detected in 23 cases (21.1\%), KRAS mutations in 13 cases (11.9\%), and $A L K$ fusion genes in 5 cases (4.9\%). The $A L K$ fusion genes could not be analyzed in four cases because of insufficient tissue samples remaining after routine histochemistry and an EGFR/KRAS mutation analysis. We found that small biopsy samples from EBUS-TBNA were adequate for performing a triple gene analysis in 97 patients (96\%). ALK fusion protein immunohistochemistry (IHC) was $100 \%$ consistent with fluorescence in situ hybridization (FISH).

Conclusion Small samples obtained by EBUS-TBNA were found to be sufficient for performing a triple gene analysis following routine histology and IHC. ALK IHC showed a very good concordance with FISH for detecting $A L K$ fusion genes.
\end{abstract}

Key words: EBUS-TBNA, lung cancer, EGFR mutations, KRAS mutations, ALK fusion genes

(Intern Med 55: 3105-3111, 2016)

(DOI: 10.2169/internalmedicine.55.6794)

\section{Introduction}

An essential part of the treatment for advanced non-small cell lung cancer (NSCLC) is therapy tailored to the particular histology and mutation status of individual patients (1). Tumor cells with somatic mutations of the tyrosine kinase domain of the epidermal growth factor receptor (EGFR) respond well to tyrosine kinase inhibitors (TKI) (2). The RAS pathway, a major signaling pathway activated by EGFR, correlates with EGFR TKI resistance in the presence of gene mutations that encode the V-Ki-ras2 Kirsten rat sarcoma vi- ral oncogene homolog (KRAS) domain (3). In addition, NSCLC incorporating a fusion gene located between the anaplastic lymphoma kinase $(A L K)$ gene and the echinoderm microtubule-associated protein-like 4 (EMLA) gene responds well to $A L K$ inhibitors such as crizotinib (4).

Fewer than $30 \%$ of patients are able to undergo diagnostic surgery for lung cancer; therefore, most diagnoses arise from small biopsies acquired by bronchoscopy or percutaneous needle aspiration (PCNA). However, these are associated with some limitations regarding mediastinal staging (5). Endobronchial ultrasound-guided transbronchial needle aspiration (EBUS-TBNA) is a minimally-invasive alternative to

${ }^{1}$ Division of Pulmonary and Critical Care Medicine, Department of Medicine, Samsung Medical Center, Sungkyunkwan University School of Medicine, Korea and ${ }^{2}$ Department of Pathology, Samsung Medical Center, Sungkyunkwan University School of Medicine, Korea Received for publication November 4, 2015; Accepted for publication March 22, 2016 Correspondence to Dr. Sang-Won Um, sangwonum@skku.edu 
mediastinoscopy for the diagnosis and staging of NSCLC (6). EBUS-TBNA can be initially performed to simultaneously diagnose and determine the mediastinal stage in cases of suspected mediastinal metastasis identified via integrated positron emission tomography/computed tomography (PET/CT). To provide treatment tailored to individual patients, an analysis of the lung cancer subtypes and genotypes, and in particular EGFR/KRAS mutations and $A L K$ fusion genes, is critical (7). Several studies that used small NSCLC tissue samples obtained via EBUS-TBNA have proved that such pathologic classification is feasible $(8,9)$. As well as accurate cell typing, EBUS-TBNA can also be used to detect EGFR and KRAS mutations in cell blocks possessing tumor cells (10), as well as $A L K$ fusion genes in metastatic lymph node samples (11). In this study, we performed simultaneous histologic classification and a mutation analysis (including EGFR, KRAS, and $A L K$ gene rearrangement) using metastatic lymph node or lung tissue samples obtained by EBUS-TBNA. The efficacy of a triple gene analysis using these samples was investigated in patients with suspected metastatic cancer of the lymph nodes or a primary lung mass near the bronchus that made it possible to carry out EBUS-TBNA tissue sampling.

\section{Materials and Methods}

\section{Patients}

Between May 2011 and May 2013, patients at the Samsung Medical Center (Seoul, South Korea) with suspected primary lung cancer were retrospectively enrolled in this study. EBUS-TBNA was performed on suspected metastatic lymph nodes or lung masses to obtain tissue samples for diagnosis and a mutational analysis. If malignant cells were histologically detected, then mutation tests for $E G F R$, $K R A S$, and EMLA-ALK were routinely performed at the discretion of the pathologist, except in cases of small cell lung cancer and extrathoracic malignancy. Most of the enrolled patients underwent chest $\mathrm{CT}$ and integrated PET/CT prior to EBUS-TBNA. The acquisition of the integrated PET/CT images was undertaken using the GE Discovery LS PET/CT system (GE Healthcare, Milwaukee, USA) consisting of a GE Advance NXi PET scanner and a GE LightSpeed Plus 8-slice CT scanner. We considered a lymph node or lung mass to be positive for malignancy if the maximum standardized uptake value $\left(\mathrm{SUV}_{\max }\right)$ was greater than 2.5 on ${ }^{18} \mathrm{~F}$ fluorodeoxy glucose (FDG) PET/CT (12). The Institutional Review Board of the Samsung Medical Center approved this study and waived the requirement for informed consent from each patient. All medical records and personnel information was anonymized and assigned to subject identification code prior to data analysis.

\section{EBUS-TBNA}

EBUS-TBNA was performed with a flexible convex probe ultrasonic puncture bronchoscope with a linear scanning transducer set to a frequency of $7.5 \mathrm{MHz}$ (CP-EBUS, BFUC206F-OL8, Olympus, Tokyo, Japan) $(6,13)$. The procedure was carried out on an inpatient basis under local anesthesia and conscious sedation with midazolam. The indications for EBUS-TBNA in the mediastinal or hilar lymph nodes were the presence of either of these lymph nodes with a short axis diameter $\geq 10 \mathrm{~mm}$ on chest CT and a lymph node with a $\mathrm{SUV}_{\max }$ greater than 2.5. EBUS-TBNA was also used to acquire lung mass tissue close to the bronchus, particularly when located in an area where PCNA would be difficult or dangerous to perform (14). All aspirate specimens were expelled onto glass slides, smeared, immediately fixed, and then sent for cytological and/or histological examination. Rapid on-site cytopathological evaluation was not carried out. Pathology with hematoxylin and eosin staining was recorded and classed as benign if it predominantly contained lymphocytes, but no evident malignant cells, or as malignant if it contained clearly malignant cells.

\section{Pathological and molecular processing of EBUS- TBNA samples}

Core samples obtained from a 22-gauge needle were processed using standard histopathologic techniques. The fresh specimen was placed in a formaldehyde solution, which acted as a fixing agent. After wax infiltration and paraffin embedding, the specimen was mounted on a slide and diagnosed histologically using immunohistochemistry (IHC). To classify lung cancer, IHC was performed using monoclonal antibodies applied to CK, p53, p56, p63, TTF-1, and $A L K$. The Novocastra mouse monoclonal antibody p80 ALK (Clone 5A4, NCL-ALK, Leica, Wetzlar, Germany) was used for the immunostaining of ALK. If lung cancer was diagnosed, then the microdissection of tumor cells was done to extract DNA from the paraffin-embedded tissue specimens. A mutational analysis of EGFR exons 18-21 and KRAS codons 12,13 , and 61 was then undertaken. Direct sequencing was utilized for mutations of the EGFR exons 18-21. Some samples were analyzed with a peptide nucleic acid (PNA)mediated real-time polymerase chain reaction (PCR) clamping method that enables the detection of EGFR mutants down to approximately $1 \%$ mutant-to-wild type, followed by direct sequencing to confirm the detection of EGFR mutations. A $K R A S$ mutational analysis was simultaneously performed with the extracted DNA using direct sequencing or PNA-mediated real-time PCR clamping in codons 12 and 13 of exon 2. The rearrangement of the $A L K$ locus on chromosome 2p23 was detected using the Vysis ALK Break-Apart fluorescence in situ hybridization (FISH) probe (Abbott Molecular, Des Plaines, USA). An expert pathologist (Choi YR) from the Department of Pathology selected samples containing sufficient tumor cells to conduct a qualified analysis. The positive criteria for EML4-ALK translocation was defined as a count of more than 50 cells and $>15 \%$ of the counted cells displaying green and red signals separated by at least two signal diameters and/or an isolated red signal. The translocation was considered to be negative if the sam- 
Table 1. Clinical Characteristics of the Patients with NSCLC.

\begin{tabular}{lr}
\hline Characteristics & Patients $(\mathrm{n}=109)$ \\
\hline Gender & $77(70.6)$ \\
Male & $32(29.4)$ \\
Female & $63.3 \pm 9.64$ \\
Age, y & \\
Histology of metastatic lymph node & $66(60.6)$ \\
$\quad$ Adenocarcinoma & $10(9.2)$ \\
Favor adenocarcinoma & $24(22.0)$ \\
Squamous cell carcinoma & $5(4.6)$ \\
Favor squamous cell carcinoma & $4(3.7)$ \\
NSCLC-NOS & $4(3.7)$ \\
Clinical stage & $50(45.9)$ \\
II & $51(46.8)$ \\
III & $4(3.7)$ \\
IV & \\
Recurrence & $40(36.7)$ \\
Smoking & $69(63.3)$ \\
Non-smoker & \\
Smoker & \\
\hline Data are presented as the means \pm SD or as $n(\%)$, unless \\
otherwise indicated. NSCLC: non-small cell lung cancer, \\
NOS: not otherwise specified
\end{tabular}

ples did not fulfil the above criteria.

\section{Statistical analysis}

Clinical characteristics and the mutation status are presented as median (range) values for continuous variables and as numbers (percentage) for categorical variables. A comparison between the EMLA-ALK fusion positive and negative groups was performed with Fisher's exact test. Concordance between the $A L K$ IHC and $A L K$ FISH methods was estimated with K-coefficients. A p value $<0.05$ was taken to indicate statistical significance based on a two-sided hypothesis. The IBM SPSS Statistics for Windows software package (ver. 22.0; IBM Corp., Armonk, USA) was used for the data analysis.

\section{Results}

\section{Clinical characteristics of patients with NSCLC}

Table 1 lists the baseline characteristics of the 109 patients included in this study. A total of 155 patients with suspected primary lung cancer underwent diagnostic EBUSTBNA. Forty-six patients were excluded prior to analysis because they were diagnosed with small cell lung cancer, benign disease, or extrathoracic metastasis, or because the results were non-diagnostic. The median aspiration number of EBUS-TBNA per node and lung mass was 2.0 (range, 15) and 3.0 (range, 2-5), respectively. The samples diagnosed to be NSCLC were processed before undergoing IHC for histological classification and gene analysis (Fig. 1). The histology of the metastatic lymph nodes or lung tissue specimens in 109 patients revealed 66 adenocarcinoma (60.6\%), 10 favor adenocarcinoma (9.2\%), 24 squamous cell carcinoma (22.0\%), 5 favor squamous cell carcinoma (4.6\%), and 4 NSCLC-not otherwise specified (3.7\%), respectively. In total, 69 patients were smokers $(63.3 \%)$, compared to 40 non-smokers $(36.7 \%)$.

\section{Mutation status of samples obtained by EBUS-TBNA}

Table 2 summarizes the mutation status of EGFR/KRAS mutations, and $A L K$ rearrangement. A total of 109 EBUSTBNA samples underwent a mutation analysis. Among the 76 patients with adenocarcinoma, EGFR mutations were interpreted as positive in 22 samples $(28.9 \%)$, KRAS mutations in $12(12.8 \%)$ samples, and $A L K$ rearrangement in 5 samples (7.1\%). Fig. 2 shows representative images regarding the pathology and mutational tests including EGFR, KRAS and $A L K$. Among those patients with squamous cell carcinoma, EGFR mutations were interpreted as positive in one sample (3.4\%); KRAS mutations were also interpreted as positive in one sample (3.4\%). However, there was no positive interpretation regarding $A L K$ rearrangements. Triple gene testing for $E G F R / K R A S$ mutations and $A L K$ rearrangement was simultaneously performed for 101 samples because the $A L K$-FISH test was not performed for 8 samples. The mean tumor cell percentage was $40 \%$ [interquartile range (IQR): 20-70] for adenocarcinoma, 30\% (IQR: 10-60) for squamous cell carcinoma, and 30\% (IQR: 20-70) for NSCLC-NOS. Sufficient samples in terms of both quantity and quality were obtained via EBUS-TBNA to enable the testing of 109 consecutive NSCLC tissue specimens. However, the $A L K$ rearrangement test was not able to be carried out in 4 out of 101 consecutive samples. There were no residual tissue blocks containing tumor cells in order to carry ou the ALK rearrangement test after the analysis of EGFR and KRAS mutation. The median duration of the entire process, from EBUS-TBNA to the final reports, was 14 days (range: 6-37 days).

\section{EML4-ALK fusion gene in NSCLC}

The frequency of lung cancer incorporating the EML4$A L K$ fusion gene was $5(4.9 \%)$ out of 101 patients, with a median age of 57.0 years. Comparison of the clinical characteristics between EML4-ALK fusion positive and negative patients is detailed in Table 3 . The smoking status of the patients with lung cancer incorporating fusion genes was significantly lower ( 0.0 pack-year, IQR $0-0.75$ ) compared to the patients with lung cancer and no $A L K$ gene rearrangement (20.0 pack year, IQR 0-40). All lung cancer cases which included the EML4-ALK fusion gene had adenocarcinoma and no positive $E G F R / K R A S$ mutations. On the other hand, lung cancer that was negative for fusion genes in $22.8 \%$ of the EGFR mutation positive cases and $11.9 \%$ of the KRAS mutations. The ALK IHC and EMLA-ALK FISH methods closely correlated with the $5 A L K$ IHC positive and $92 A L K$ negative cases. All $A L K$ IHC positive cases revealed $E M L 4-A L K$ fusion at the $A L K$ locus on chromosome 2p23.

\section{Discussion}

This study showed that the EBUS-TBNA procedure could 


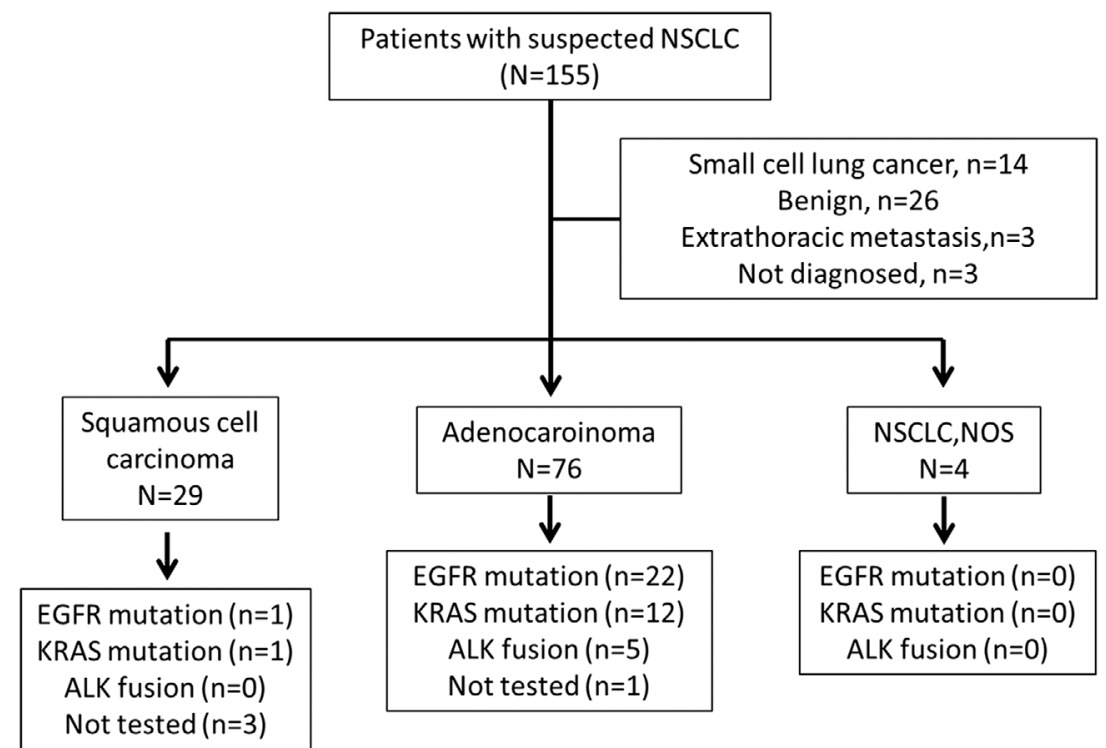

Figure 1. Diagnostic algorithm of the study patients. EGFR: epidermal growth factor receptor, KRAS: V-Ki-ras2 Kirsten rat sarcoma viral oncogene homolog, ALK: anaplastic lymphoma kinase, NSCLC: non-small cell lung cancer

Table 2. Mutation Status According to the Histologic Subtyping.

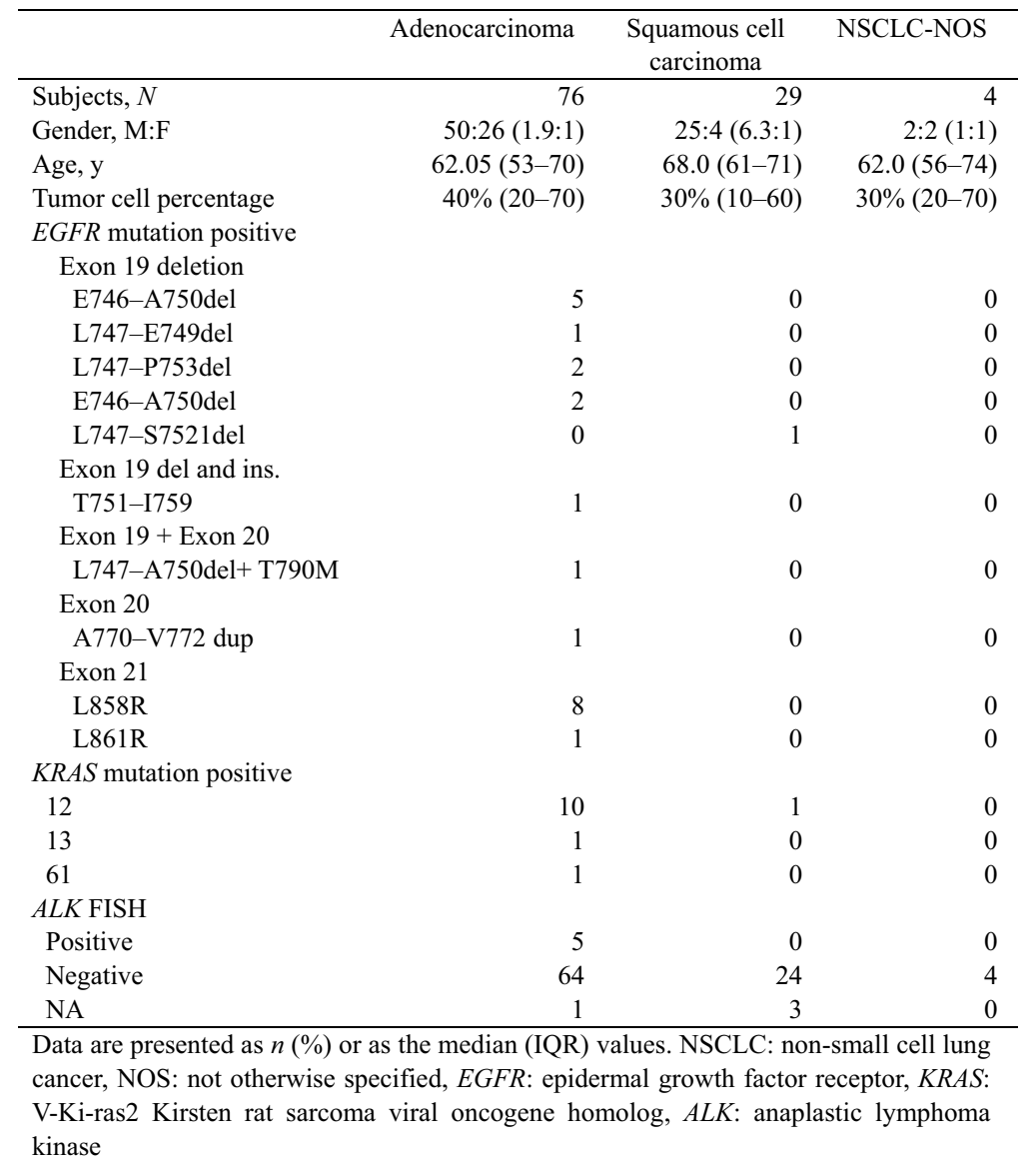

provide sufficient aspiration samples for $100 \%$ (EGFR and KRAS mutations) and in $96 \%$ (ALK gene rearrangement) of the enrolled patients when they were processed consecutively. A routine triple gene analysis following pathologic subtyping was feasible in $96 \%$ of our study samples. The
EGFR gene is frequently expressed in NSCLC and somatic mutations in exons 18-21 have been shown to demonstrate a relationship with the treatment responses to EGFR TKI (2). EML4-ALK rearrangement should also be identified to predict the response from the $A L K$ inhibitor (15). Such genetic 

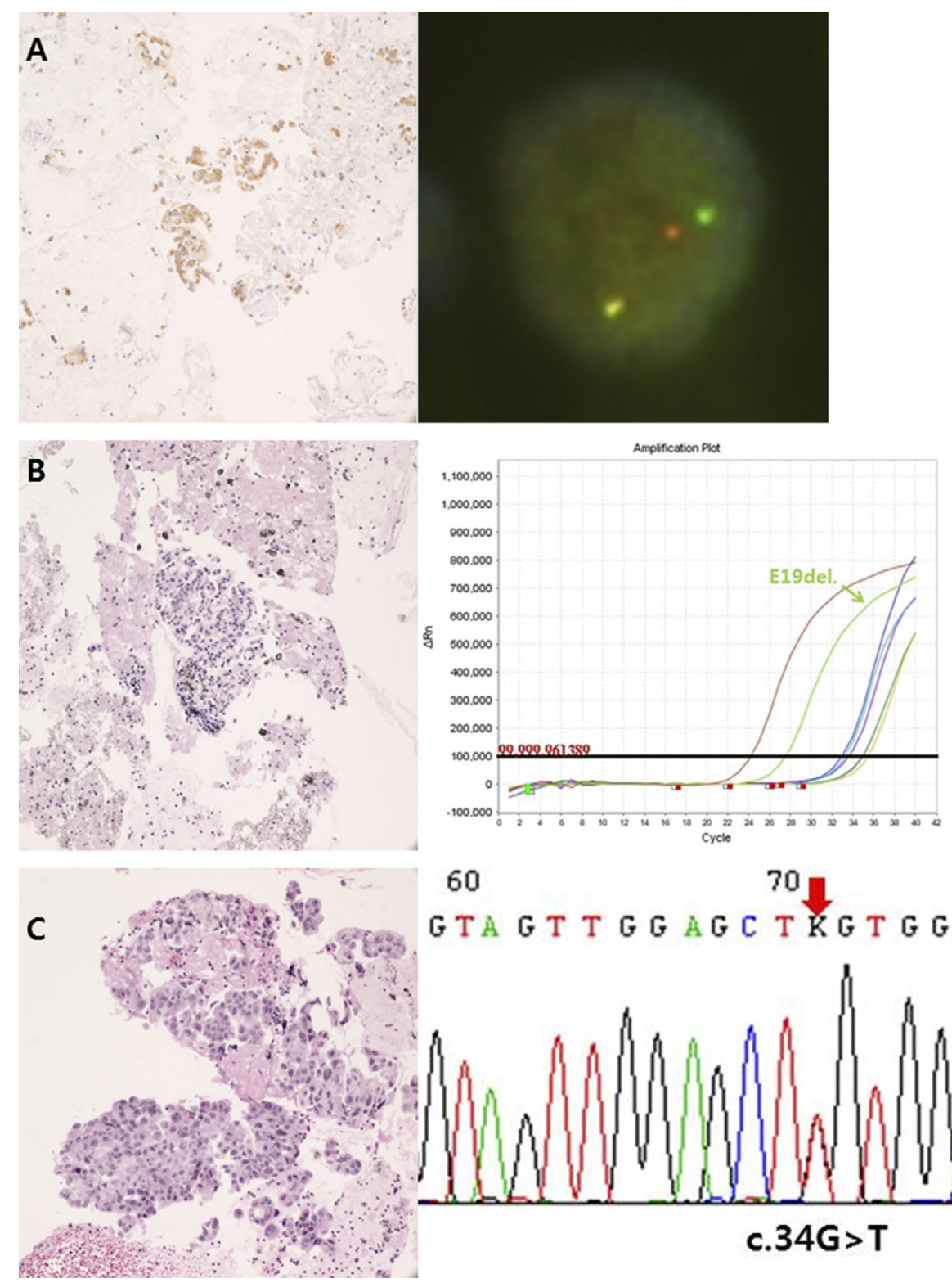

60

GTA GT T G G A G C T K G T G G C GTA G C Aे मे

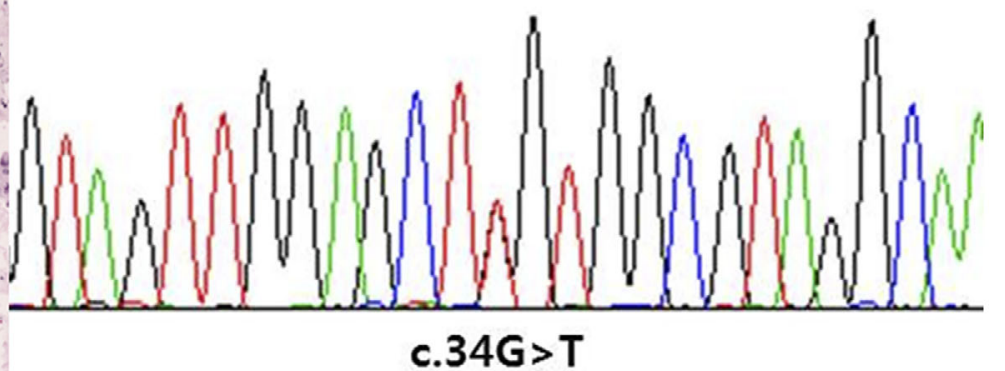

Figure 2. Representative images of the samples obtained by EBUS-TBNA. A, Carcinoma cells are positive for $A L K$ with a membranous pattern of staining. The $A L K$ fluorescence in situ hybridization test shows break apart rearrangement in 40 of 50 non-overlapping nuclei: one red, one green, and one fusion signal. B, Metastatic adenocarcinoma cells occupy about 5\% of total specimen volume. Realtime polymerase chain reaction test detects a deletion mutation in exon 19: c.2235_2,249 del 15 (p. E746_A750 del); The standard cyclic threshold (Ct) is 34 and sample $\mathrm{Ct}$ is 27.5436. C, Metastatic adenocarcinoma cells occupy about $70 \%$ of the total specimen volume. KRAS sequencing shows a missense mutation in the 12th codon: c.34G > T (p.G12C).

information is a key to providing personalized treatments for patients with advanced NSCLC. Previous studies regarding gene analyses for EGFR and KRAS concluded that the use of EBUS-TBNA in advanced lung cancer allowed for the adequate interpretation of mutations (16). However, the quality of such interpretation using samples obtained by EBUS-TBNA has only rarely been investigated. Folch et al. described the success and failure rate of EGFR mutations and $A L K$ translocations from nodal tissue specimens using EBUS-TBNA and other diagnostic methods. EBUS-TBNA was able to be performed in 42 out of 207 cases, resulting in a success rate of $95.2 \%$ in EGFR mutations, $90.5 \%$ in KRAS mutations, and $90.5 \%$ in $A L K$ FISH (17). In contrast, our study used samples obtained via EBUS-TBNA alone, and a total of 109 cases were enrolled providing a high success rate of $100 \%$ in EGFR/KRAS mutations and $96 \%$ in $A L K$ translocations. The $A L K$ translocation rate for adenocarcinoma was $7.1 \%$. There was no overlap between $E G F R$, $K R A S$, and $A L K$ mutations. Pathologist-interpreted $A L K$ immunohistochemistry was performed in all NSCLC cases. The concordance rate of $A L K$ IHC in the small samples obtained by EBUS-TBNA was perfectly matched in our study. 
Table 3. Comparison of the Patient Characteristics Between EML4-ALK Fusion Positive and Negative NSCLC.

\begin{tabular}{|c|c|c|c|}
\hline \multirow{2}{*}{ Characteristics } & \multicolumn{2}{|c|}{$E M L 4-A L K$ fusion } & \multirow{2}{*}{$\mathrm{p}$ value } \\
\hline & Positive $(n=5)$ & Negative $(n=92)$ & \\
\hline Age & $57.0(49-63)$ & $64.0(55-71)$ & 0.096 \\
\hline Gender, Female & $3(60)$ & $28(30.4)$ & 0.087 \\
\hline Smoking status, PY & $0.0(0-0.75)$ & $20.0(0-40)$ & 0.023 \\
\hline \multicolumn{4}{|l|}{ Metastasis } \\
\hline Brain & $0(0.0)$ & $9(9.8)$ & 0.404 \\
\hline Bone & $0(0.0)$ & $15(16.3)$ & 0.616 \\
\hline Pleura & $1(20.0)$ & $20(21.7)$ & 0.962 \\
\hline Histology of LN & & & 0.739 \\
\hline Adenocarcinoma & $5(100)$ & $64(69.6)$ & \\
\hline SQC & $0(0.0)$ & $24(26.0)$ & \\
\hline NSCLC-NOS & $0(0.0)$ & $4(4.3)$ & \\
\hline$A L K \mathrm{IHC}$ & & & 0.000 \\
\hline Positive & $5(100)$ & $0(0.0)$ & \\
\hline Negative & $0(0.0)$ & $92(100)$ & \\
\hline$E G F R$ mutation status & & & 0.440 \\
\hline Positive & $0(0.0)$ & $21(22.8)$ & \\
\hline Negative & $5(100)$ & $71(77.2)$ & \\
\hline$K R A S$ mutation status & & & 0.627 \\
\hline Positive & $0(0.0)$ & $11(12.0)$ & \\
\hline Negative & $5(100)$ & $81(88.0)$ & \\
\hline \multicolumn{4}{|c|}{$\begin{array}{l}\text { Data are presented as } n(\%) \text { or as the median (IQR) values. PY: pack-year, LN: } \\
\text { lymph node, SQC: squamous cell carcinoma, IHC: immunohistochemistry, } \\
E M L 4-A L K \text { : echinoderm microtubule-associated protein-like 4-anaplastic } \\
\text { lymphoma kinase }\end{array}$} \\
\hline
\end{tabular}

This result shows that $A L K$ translocation IHC could be employed as a screening method for $A L K$ rearrangement prior to $A L K$ FISH in EBUS-TBNA-sampled tissues.

EBUS-TBNA is a non-invasive procedure performed under conscious sedation to diagnose cancer of the mediastinal and hilar lymph nodes. Our institute has issued a protocol to reduce the time interval from the initial biopsy to the pathologic diagnosis and interpretation of gene mutations, since treatment delays affect the prognosis of advanced lung cancer (18). EBUS-TBNA can be performed as an initial procedure for simultaneous diagnosis and mediastinal staging if PET/CT reveals a significant mediastinal lymph node uptake in a patient with suspected lung cancer. In this study, we demonstrated that small samples obtained by EBUSTBNA provided good quality subtyping for NSCLC with mediastinal metastasis. Of those patients diagnosed with NSCLC in our study, only $3.7 \%$ had NSCLC-NOS. The most common pathologic NSCLC classifications was adenocarcinoma $(60.6 \%)$ and favor adenocarcinoma (9.2\%), followed by squamous cell carcinoma (22\%), and favor squamous cell carcinoma (4.6\%). From the IHC results, $96.3 \%$ of the sampled tissues were classified by the time lung cancer was pathologically diagnosed. A multicenter study of 774 patients from the UK suggested that small samples obtained via EBUS-TBNA could be used to classify the NSCLC subtypes in $77 \%$ of cases (8). However, this study was limited in that it did not perform routine IHC. Our data indicate a higher rate of lung cancer subtypes because we routinely performed IHC in all tissues obtained by EBUS-TBNA. The median time lapse between EBUSTBNA and the final reports, including the mediastinal stage, tumor diagnosis with pathologic subtype, and genetic infor- mation (EGFR/KRAS mutations, and $A L K$ rearrangement) was 14 days (range: 6-37 days). Past practice was to request a mutational analysis after the patient had been diagnosed with lung cancer via bronchoscopy or PCNA. Following diagnosis, EBUS-TBNA was then performed for mediastinal staging when the patient showed a significant mediastinal uptake on PET/CT. However, this led to delays in treatment because clinicians required information on the mediastinal stage, pathologic subtype, and mutation status before any decisions on treatment could be made. The protocol in place at our institute requires EBUS-TBNA to be performed initially to confirm the lung cancer staging or diagnosis, pathologic subtype, and genetic status of EGFR, KRAS, and $E M L 4-A L K$ in patients suspected of having advanced lung cancer following PET/CT.

Our study is associated with some limitations. A recent analysis of the data from a large number of patients has reported that $A L K$ rearrangements are mutually exclusive, with mutations in either EGFR or KRAS (19). Therefore, to be cost-effective, EML4-ALK fusion gene assessment should not be undertaken in samples harboring EGFR mutations. Second, the $A L K$ IHC results were expressed as either positive or negative, and a scoring system according to IHC expression was not adopted. At the time we conducted our study there was little evidence that $A L K$ IHC was a useful screening tool for $A L K$ gene rearrangement. We also analyzed $A L K$ gene arrangement despite the presence of positive $A L K$ IHC cases. Third, we analyzed multiple genotypes using direct sequencing or a PNA-mediated real-time PCR clamping method. The sensitivity of these two methods in detecting EGFR/KRAS mutations may differ. Further studies using the PNA-mediated real-time PCR clamping method 
may be required to validate this suggestion. In addition, the $A L K$ FISH results using small biopsy samples from EBUSTBNA may differ from those obtained with excision samples. Fourth, there was no information of the comparison data from samples obtained by transbronchial lung biopsy (TBLB) for the triple mutation analysis. The use of EBUSTBNA samples may be further supported if we compared the detection rate of triple mutations from samples obtained between EBUS-TBNA and TBLB.

In conclusion, small tissue samples obtained via EBUSTBNA were found to be sufficient to successfully detect triple mutations in advanced lung cancer. This demonstrates the usefulness of EBUS-TBNA as an initial modality with regard to the treatment of lung cancer.

The authors state that they have no Conflict of Interest (COI).

\section{References}

1. Buettner R, Wolf J, Thomas RK. Lessons learned from lung cancer genomics: the emerging concept of individualized diagnostics and treatment. J Clin Oncol 31: 1858-1865, 2013.

2. Rosell R, Carcereny E, Gervais R, et al. Erlotinib versus standard chemotherapy as first-line treatment for European patients with advanced EGFR mutation-positive non-small-cell lung cancer (EURTAC): a multicentre, open-label, randomised phase 3 trial. Lancet Oncol 13: 239-246, 2012.

3. Eberhard DA, Johnson BE, Amler LC, et al. Mutations in the epidermal growth factor receptor and in KRAS are predictive and prognostic indicators in patients with non-small-cell lung cancer treated with chemotherapy alone and in combination with erlotinib. J Clin Oncol 23: 5900-5909, 2005.

4. Kwak EL, Bang YJ, Camidge DR, et al. Anaplastic lymphoma kinase inhibition in non-small-cell lung cancer. N Engl J Med 363: 1693-1703, 2010.

5. Takeshita J, Masago K, Kato R, et al. CT-guided fine-needle aspiration and core needle biopsies of pulmonary lesions: a singlecenter experience with 750 biopsies in Japan. AJR Am J Roentgenol 204: 29-34, 2015.

6. Um SW, Kim HK, Jung SH, et al. Endobronchial ultrasound versus mediastinoscopy for mediastinal nodal staging of non-smallcell lung cancer. J Thorac Oncol 10: 331-337, 2015.

7. Gaughan EM, Costa DB. Genotype-driven therapies for non-small cell lung cancer: focus on EGFR, KRAS and ALK gene abnormalities. Ther Adv Med Oncol 3: 113-125, 2011.

8. Navani N, Brown JM, Nankivell M, et al. Suitability of endobronchial ultrasound-guided transbronchial needle aspiration specimens for subtyping and genotyping of non-small cell lung cancer: a multicenter study of 774 patients. Am J Respir Crit Care Med 185: 1316-1322, 2012.

9. Esterbrook G, Anathhanam S, Plant PK. Adequacy of endobronchial ultrasound transbronchial needle aspiration samples in the subtyping of non-small cell lung cancer. Lung Cancer 80: 30-34, 2013.

10. Schuurbiers OC, Looijen-Salamon MG, Ligtenberg MJ, van der Heijden HF. A brief retrospective report on the feasibility of epidermal growth factor receptor and KRAS mutation analysis in transesophageal ultrasound- and endobronchial ultrasoundguided fine needle cytological aspirates. J Thorac Oncol 5: 1664$1667,2010$.

11. Neat MJ, Foot NJ, Hicks A, et al. ALK rearrangements in EBUSderived transbronchial needle aspiration cytology in lung cancer. Cytopathology 24: 356-364, 2013.

12. Khalaf M, Abdel-Nabi H, Baker J, Shao Y, Lamonica D, Gona J. Relation between nodule size and 18F-FDG-PET SUV for malignant and benign pulmonary nodules. J Hematol Oncol 1: 13, 2008.

13. Jhun BW, Park HY, Jeon K, et al. Nodal stations and diagnostic performances of endobronchial ultrasound-guided transbronchial needle aspiration in patients with non-small cell lung cancer. J Korean Med Sci 27: 46-51, 2012.

14. Verma A, Jeon K, Koh WJ, et al. Endobronchial ultrasound-guided transbronchial needle aspiration for the diagnosis of central lung parenchymal lesions. Yonsei Med J 54: 672-678, 2013.

15. Shaw AT, Yeap BY, Solomon BJ, et al. Effect of crizotinib on overall survival in patients with advanced non-small-cell lung cancer harbouring ALK gene rearrangement: a retrospective analysis. Lancet Oncol 12: 1004-1012, 2011.

16. Nakajima T, Yasufuku K, Nakagawara A, Kimura H, Yoshino I. Multigene mutation analysis of metastatic lymph nodes in nonsmall cell lung cancer diagnosed by endobronchial ultrasoundguided transbronchial needle aspiration. Chest 140: 1319-1324, 2011.

17. Folch E, Yamaguchi N, VanderLaan PA, et al. Adequacy of lymph node transbronchial needle aspirates using convex probe endobronchial ultrasound for multiple tumor genotyping techniques in nonsmall-cell lung cancer. J Thorac Oncol 8: 1438-1444, 2013.

18. Gomez DR, Liao KP, Swisher SG, et al. Time to treatment as a quality metric in lung cancer: Staging studies, time to treatment, and patient survival. Radiother Oncol 115: 257-263, 2015.

19. Gainor JF, Varghese AM, Ou SH, et al. ALK rearrangements are mutually exclusive with mutations in EGFR or KRAS: an analysis of 1,683 patients with non-small cell lung cancer. Clin Cancer Res 19: 4273-4281, 2013.

The Internal Medicine is an Open Access article distributed under the Creative Commons Attribution-NonCommercial-NoDerivatives 4.0 International License. To view the details of this license, please visit (https://creativecommons.org/licenses/ by-nc-nd/4.0/).

(C) 2016 The Japanese Society of Internal Medicine http://www.naika.or.jp/imonline/index.html 